\title{
AVALIAÇÃO DO AUTOCUIDADO NOS PORTADORES DE INSUFICIÊNCIA CARDÍACA
}

\author{
Juliana Medeiros ${ }^{1}$, Carolina de Araújo Medeiros $^{2}$
}

\begin{abstract}
RESUMO: O objetivo deste estudo foi caracterizar o perfil clínico e sociodemográfico dos portadores de Insuficiência Cardíaca e descrever o comportamento de autocuidado. Estudo transversal descritivo, com amostra não probabilística de 57 pacientes da enfermaria cardiológica de hospital público do Recife, Brasil, entre maio e setembro de 2016. O autocuidado foi avaliado pela Self-Care of Heart Failure Index versão 6.2, versão brasileira. A idade média dos participantes foi de 62,9 anos; 31 (54,4\%) homens; $36(63,2 \%)$ alfabetizados; $26(45,6 \%)$ de etiologia hipertensiva e $30(52,6 \%)$ em classe funcional III. As médias dos escores nas subescalas indicaram autocuidado inadequado (manutenção do autocuidado: 43,3; manejo do autocuidado: 37,5 e confiança do autocuidado: 54,3). Escores mais elevados foram associados com escolaridade, os alfabetizados obtiveram média de 61 pontos $(p=0,002)$. São necessários investimentos para melhorar o autocuidado e a enfermagem pode ter papel relevante nessa melhora.
\end{abstract}

DESCRITORES: Autocuidado; Insuficiência cardíaca; Enfermagem cardiovascular; Qualidade de vida; Doença crônica.

\section{ASSESSMENT OF SELF-CARE IN HEART FAILURE PATIENTS}

\begin{abstract}
The objective in this study was to characterize the clinical and sociodemographic profile of heart failure patients and to describe the self-care behavior. Descriptive and cross-sectional study with a non-probabilistic sample of 57 cardiology nursing patients at a public hospital in Recife, Brazil, between May and September 2016. The self-care was assessed by means of the Self-Care of Heart Failure Index version 6.2, Brazilian version. The participants' mean age was 62.9 years; 31 (54.4\%) men; 36 (63.2\%) literate; $26(45.6 \%)$ suffering from hypertensive etiology and $30(52.6 \%)$ in functional class III. The mean scores for the subscales indicated inappropriate self-care (self-care maintenance: 43.3; self-care management: 37.5 and confidence in self-care: 54.3 ). Higher scores were associated with education, with literate participants obtaining a mean score of 61 points $(\mathrm{p}=0.002)$. Investments are needed to improve self-care and nursing can play a relevant role in this improvement.
\end{abstract}

DESCRIPTORS: Self-care; Heart failure; Cardiovascular nursing; Quality of life: Chronic illness.

\section{EVALUACIÓN DEL AUTOCUIDADO EN LOS PORTADORES DE INSUFICIENCIA CARDÍACA}

RESUMEN: El objetivo de este estudio fue caracterizar el perfil clínico y sociodemográfico de los portadores de Insuficiencia Cardíaca y describir el comportamiento de autocuidado. Estudio transversal descriptivo, con muestra no probabilística de 57 pacientes de la enfermaría cardiológica de hospital público de Recife, Brasil, entre mayo y septiembre de 2016. El autocuidado fue evaluado por la Self-Care of Heart Failure Index versión 6.2, versión brasileña. El promedio de edad de los participantes fue de 62,9 años; 31 $(54,4 \%)$ hombres; 36 (63,2\%) alfabetizados; 26 (45,6\%) de etiología hipertensiva y 30 (52,6\%) en clase funcional III. Los promedios de los scores en las subescalas indicaron autocuidado inadecuado (mantenimiento del autocuidado: 43,3; gestión del autocuidado: 37,5 y confianza en el autocuidado: 54,3 ). Puntuaciones superiores fueron asociadas con escolaridad, los alfabetizados alcanzaron promedio de 61 puntos $(p=0,002)$. Son necesarias inversiones para mejorar el autocuidado y la enfermería puede ter papel relevante en esa mejora.

DESCRIPTORES: Autocuidado; Insuficiencia cardíaca; Enfermería cardiovascular; Calidad de vida; Enfermedad crónica.

${ }^{1}$ Enfermeira. Especialista em Cardiologia. Residente de Enfermagem em Cardiologia no Hospital Dom Helder Câmara. Cabo de Santo Agostinho, PE, Brasil.

${ }^{2}$ Enfermeira. Mestre em Ciências da Saúde. Enfermeira Assistencial da Secretaria de Saúde de Pernambuco. Recife, PE, Brasil. 


\section{INTRODUÇÃO}

A Insuficiência Cardíaca (IC) é uma síndrome crônica e progressiva, associada a readmissões hospitalares, baixa qualidade de vida, risco de mortalidade precoce, e geradora de altos custos para o sistema de saúde. É uma doença de caráter sistêmico, definida como disfunção cardíaca que ocasiona inadequado suprimento sanguíneo para atender necessidades metabólicas tissulares, na presença de retorno venoso normal, ou fazê-lo somente com elevadas pressões de enchimento. Evidenciada por um conjunto de sinais e sintomas de congestão pulmonar e sistêmica, é caracterizada como o último estágio de todas as doenças cardiovasculares ${ }^{(1)}$.

No Brasil, estima-se que 6,4 milhões de brasileiros sofram de IC. Essa doença é responsável por altos custos hospitalares, por grande parte dos atendimentos de emergência, provoca perda da qualidade de vida e, muitas vezes, aposentadorias precoces com altos custos para o país ${ }^{(2)}$.

O tratamento da IC é constituído pela associação da terapia farmacológica e não farmacológica. O tratamento farmacológico consiste na otimização de fármacos com impacto comprovado em morbidade e mortalidade. Associado ao tratamento farmacológico, medidas relacionadas à adesão às medicações e mudanças no estilo de vida relacionadas ao autocuidado trazem benefícios comprovados ${ }^{(3)}$.

O sucesso do tratamento depende da adesão do paciente, que precisa modificar comportamentos e estilos de vida. Para isso, é necessário conhecimento adequado sobre a patologia, sinais e sintomas de descompensação e seu tratamento, a fim de evitar a internação hospitalar e melhorar a qualidade de $\operatorname{vida}^{(3)}$.

$\mathrm{Na} I \mathrm{C}$, autocuidado refere-se aos comportamentos das pessoas para manter a sua saúde e às decisões que tomam sobre a piora dos sintomas quando eles ocorrem. A manutenção do autocuidado envolve a adesão às recomendações farmacológicas, consumo de dieta com pouco sal, cessação do uso de tabaco, consumo limitado de álcool, monitoração diária do peso e de sinais ou sintomas de descompensação da IC. Nessa perspectiva, autocuidado é um processo de tomada de decisão que os pacientes usam na escolha de comportamentos que mantêm a estabilidade fisiológica, e a resposta aos sintomas quando eles ocorrem ${ }^{(4)}$.

Estudos mostram que o autocuidado na IC é inadequado e que indivíduos que participaram em programa educacional de autogestão apresentaram melhor comportamento de autocuidado. Características sociodemográficas, aspectos culturais, nível de educação e clínicas próprias da IC são descritas na literatura como variáveis que influenciam no autocuidado de pacientes com IC ${ }^{(4)}$.

O pouco conhecimento sobre a IC e seu tratamento, bem como medidas de autocuidado, tem sido considerado preditor de instabilidade clínica e consequente readmissão hospitalar. Estudos ressaltam que as readmissões hospitalares poderiam ser evitadas em aproximadamente $40-59 \%$ dos pacientes com IC, mediante planejamento de alta, reabilitação adequada, identificação de problemas referentes ao uso correto de medicamentos e orientação do paciente e familiar para o reconhecimento precoce dos sinais e sintomas de descompensação da $I C^{(5)}$.

Acredita-se que a falta de conhecimento contribui para piora da qualidade de vida, isolamento social, aumento das comorbidades, ausência do autocuidado, desconhecimento de sinais e sintomas e a não aderência ao tratamento. Tais fatores são inerentes aos crescentes gastos com saúde, visto que o conhecimento dos pacientes sobre sua condição é determinante para a aderência e o sucesso de seu tratamento ${ }^{(6)}$.

O comportamento de autocuidado ainda é pouco estudado no Brasil, o que justifica este estudo. Conhecer o hábito dos brasileiros com IC contribui para que os enfermeiros reconheçam comportamentos que precisam ser modificados ou incluídos, para o controle da doença e consequente melhor qualidade de vida.

Diante do exposto, os objetivos deste estudo foram: caracterizar o perfil clínico e sociodemográfico dos portadores de IC e descrever o comportamento de autocuidado de pacientes adultos com IC, por meio da escala Self-Care of Heart Failure Index-SCHFI, versão brasileira. 
Estudo observacional transversal, realizado com dados obtidos por meio de entrevista presencial, composto de amostra não probabilística com 57 pacientes adultos com diagnóstico prévio de IC, internados na enfermaria cardiológica de um hospital público especializado em cardiologia, na região metropolitana do Recife, Brasil. O estudo transcorreu no período de maio a setembro de 2016.

Para o recrutamento, os pesquisadores verificavam previamente o prontuário dos pacientes potencialmente elegíveis. Com os seguintes critérios de inclusão: portadores com idade $\geq 18$ anos; de ambos os sexos, que tenham o diagnóstico de IC; segunda ou outra internação; causa do internamento ter sido IC descompensada; o paciente estar estável. Foram excluídos os pacientes de primeira internação; paciente em estado comprometido ou grave; não ter o diagnóstico de IC confirmado.

O comportamento de autocuidado foi avaliado utilizando a versão brasileira do Self-Care of Heart Failure Index $(\mathrm{SCHFI})^{(7)}$. O SCHFI é uma medida de autocuidado composta por 22 itens divididos em três escalas: manutenção do autocuidado (10 itens) relacionada aos comportamentos para manter a estabilidade fisiológica; manejo do autocuidado ( 6 itens) relacionada ao comportamento dos pacientes quando ocorrem os sintomas e confiança no autocuidado (6 itens). Os escores para cada domínio variam de 0-100 pontos, calculados a partir da transformação dos escores puros (cada item da escala varia de 1-4). Pontuações mais altas refletem autocuidado superior e escores $\geq 70$ pontos para cada subescala indicam autocuidado adequado(7).

Quanto às variáveis sociodemográficas, foram consideradas idade, sexo, escolaridade, raça, convivência marital e renda familiar. As variáveis clínicas estudadas foram: classe funcional da IC (conforme avaliação dos pesquisadores no momento da coleta de dados, seguindo a diretriz estabelecida pela New York Heart Association - NYHA) ${ }^{(6)}$ e etiologia da IC (conforme registro em prontuário do paciente).

Os dados foram analisados no software IBM ${ }^{\circledast}$ SPSS $^{\circledast}$ v. 24.0 para Microsoft Windows ${ }^{\circledast}$. Foi realizada estatística descritiva das variáveis sociodemográficas. As variáveis da Self-Care Heart Failure Index foram apresentadas por média, desvio padrão e intervalo de confiança a $95 \%$. Por fim, foi realizado análise de diferença de médias entre o escore das subescalas da Self-Care Heart Failure Index e as variáveis gênero, escolaridade, classe funcional e a etiologia da insuficiência cardíaca. A escolaridade foi dicotomizada em alfabetizado e não alfabetizado. A classe funcional foi analisada em quatro categorias e a etiologia foi analisada em seis categorias, que apareceram na pesquisa.

As variáveis dicotômicas (gênero e escolaridade) foram analisadas por meio do teste $t$-Student para amostras independentes. A classe funcional foi analisada por meio da ANOVA e a etiologia por meio do teste Kruskal-Wallis. Para todos os testes foram considerados valores estatisticamente significativos p-valor $<0,05$.

O estudo foi aprovado pelo Comitê de Ética em Pesquisa do Instituto de Medicina Integral Professor Fernando Figueira - IMIP/PE, sob parecer número 1.541.090.

\section{RESULTADOS}

Foram avaliados 57 pacientes, com idade média de 62 anos, variando de 29 a 89 anos, predominância do gênero masculino, etiologia hipertensiva e classe funcional III. Na Tabela 1 apresentam-se as características sociodemográficas e clínicas dos participantes.

Tabela 1 - Caracterização sociodemográfica e clínica dos participantes (N=57). Região Metropolitana do Recife, PE, Brasil, 2016 (continua)

\begin{tabular}{lcccc} 
Variáveis & Média & DP & N & $\%$ \\
\hline Idade (anos) & 62,9 & 12,2 & & \\
\hline
\end{tabular}




\begin{tabular}{|c|c|c|}
\hline \multicolumn{3}{|l|}{ Gênero } \\
\hline Masculino & 31 & 54,4 \\
\hline Feminino & 26 & 45,6 \\
\hline \multicolumn{3}{|l|}{ Raça } \\
\hline Branca & 16 & 28,1 \\
\hline Negro & 5 & 8,8 \\
\hline Pardo & 36 & 63,2 \\
\hline \multicolumn{3}{|l|}{ Escolaridade } \\
\hline Analfabeto & 21 & 36,8 \\
\hline Alfabetizado & 36 & 63,2 \\
\hline \multicolumn{3}{|l|}{ Estado Marital } \\
\hline Solteiro & 15 & 27,3 \\
\hline Casado & 29 & 52,7 \\
\hline Divorciado & 5 & 9,1 \\
\hline Viúvo & 6 & 10,9 \\
\hline \multicolumn{3}{|l|}{ Renda Familiar } \\
\hline$<1$ salário mínimo & 7 & 12,3 \\
\hline 1 salário mínimo & 43 & 75,4 \\
\hline$>1$ salário mínimo & 7 & 12,3 \\
\hline \multicolumn{3}{|l|}{ Etiologia } \\
\hline Hipertensiva & 26 & 45,6 \\
\hline Chagásica & 2 & 3,5 \\
\hline Isquêmica & 10 & 17,5 \\
\hline Idiopática & 13 & 22,8 \\
\hline Valvar & 3 & 5,3 \\
\hline Alcoólica & 3 & 5,3 \\
\hline \multicolumn{3}{|l|}{ Classe Funcional } \\
\hline $\mathrm{I}$ & 6 & 10,5 \\
\hline II & 16 & 28,1 \\
\hline III & 30 & 52,6 \\
\hline IV & 5 & 8,8 \\
\hline
\end{tabular}

Na Tabela 2 apresentam-se as estatísticas descritivas para as escalas manutenção do autocuidado (manutenção), manejo do autocuidado (manejo) e confiança no autocuidado (confiança). As proporções de pacientes com autocuidado adequado (escore $\geq 70$ pontos) foram: manutenção $=$ dois pacientes $(3,5 \%)$, manejo $=$ oito pacientes $(14 \%)$ e confiança $=15(26,3 \%)$.

Tabela 2 - Estatística descritiva da Self-Care Heart Failure Index, versão brasileira ( $\mathrm{N}=57)$. Região Metropolitana do Recife, PE, Brasil, 2016 (continua)

\begin{tabular}{lccc} 
& Média & $\begin{array}{c}\text { Desvio } \\
\text { Padrão }\end{array}$ & IC 95\% \\
\hline Manutenção do autocuidado & & & \\
\hline $1 . \quad$ Você se pesa? & 1,8 & 1 & $1,5-2$ \\
\hline 2. $\quad$ Verifica se os seus tornozelos estão inchados? & 2,3 & 1,3 & $1,9-2,6$ \\
\hline $\begin{array}{l}\text { 3. } \quad \text { Tenta evitar ficar doente (por exemplo: vacinar-se contra a gripe, evitar } \\
\text { contato com pessoas doentes)? }\end{array}$ & 2,6 & 1,3 & $2,3-3$ \\
\hline $4 . \quad$ Realiza alguma atividade física? & 1,4 & 0,9 & $1,1-1,6$ \\
\hline \hline
\end{tabular}




\begin{tabular}{|c|c|c|c|}
\hline 5. $\quad$ É assíduo nas consultas com médico ou enfermeiro? & 3,3 & 1,1 & $3,0-3,6$ \\
\hline 6. Ingere uma dieta com pouco sal? & 2,7 & 1,3 & $2,4-3,1$ \\
\hline 7. $\quad$ Exercita-se por 30 minutos? & 1,5 & 1 & $1,2-1,7$ \\
\hline 8. $\quad$ Esquece-se ou deixa de tomar algum de seus medicamentos? & 1,8 & 1,2 & $1,5-2,2$ \\
\hline 9. Solicita alimentos com pouco sal quando come fora ou visita alguém? & 2 & 1,3 & $1,6-2,3$ \\
\hline $\begin{array}{l}\text { 10. Usa um sistema (caixa de comprimidos ou lembretes) para ajudá-lo a } \\
\text { lembrar de seus medicamentos? }\end{array}$ & 2,5 & 1,4 & $2,1-2,9$ \\
\hline Escore total - Manutenção do autocuidado & 43,3 & 2,5 & $38,3-48,2$ \\
\hline \multicolumn{4}{|l|}{ Manejo do autocuidado } \\
\hline $\begin{array}{l}\text { 11. Com que rapidez você os reconheceu um sintoma de insuficiência } \\
\text { cardíaca? }\end{array}$ & 1,6 & 1,6 & $1,1-2$ \\
\hline 12. Reduzir o sal na sua dieta & 1,7 & 1,2 & $1,4-2$ \\
\hline 13. Reduzir a ingestão de líquidos & 1,6 & 1 & $1,3-1,8$ \\
\hline 14. Ingerir um diurético a mais & 1,2 & 0,6 & $1,0-1,3$ \\
\hline 15. Contatar seu médico ou enfermeiro para orientação & 3,1 & 1 & $2,8-3,4$ \\
\hline $\begin{array}{l}\text { 16. Pense em um dos recursos acima que você tentou na última vez em que } \\
\text { teve dificuldade para respirar ou tornozelos inchados, você tem certeza de } \\
\text { que este recurso o ajudou? }\end{array}$ & 2,5 & 1,4 & $2,1-2,8$ \\
\hline Escore total - Manejo do autocuidado & 37,5 & 3,1 & $31,4-43,9$ \\
\hline \multicolumn{4}{|l|}{ Confiança no autocuidado } \\
\hline 17. Estar livre dos sintomas de insuficiência cardíaca & 1,7 & 0,9 & $1,4-1,9$ \\
\hline 18. Seguir o tratamento recomendado & 3,2 & 0,9 & $2,9-3,4$ \\
\hline 19. Avaliar a importância de seus sintomas & 2,6 & 1 & $2,4-2,9$ \\
\hline 20. Reconhecer alterações na saúde caso elas ocorram & 2,7 & 1 & $2,5-3$ \\
\hline 21. Fazer algo que possa aliviar seus sintomas & 2,8 & 0,9 & $2,6-3$ \\
\hline 22. Avaliar se um medicamento funciona & 2,8 & 0,9 & $2,6-3$ \\
\hline Escore total - Confiança no autocuidado & 54,3 & 2,8 & $48,6-60$ \\
\hline
\end{tabular}

As Tabelas 3, 4 e 5 apresentam os resultados dos testes de associação entre variáveis selecionadas e a SCHFI. Na subescala confiança no autocuidado, a variável escolaridade $(p=0,002)$ teve significância para um melhor autocuidado (Tabela 5).

Tabela 3 - Análise de diferença de médias entre as variáveis selecionadas e a Self-Care Heart Failure Index, subescala manutenção do autocuidado, versão brasileira ( $N=57)$. Região Metropolitana do Recife, PE, Brasil, 2016 (continua)

\begin{tabular}{|c|c|c|c|}
\hline Variáveis & Média & DP & p-valor \\
\hline Gênero & & & 0,46 \\
\hline Masculino & 45 & 20,7 & \\
\hline Feminino & 41,3 & 16 & \\
\hline Escolaridade & & & 0,12 \\
\hline Analfabeto & 38,2 & 19,2 & \\
\hline Alfabetizado & 46,2 & 17,9 & \\
\hline Classe Funcional & & & 0,466 \\
\hline 1 & 37,8 & 13,9 & \\
\hline II & 47,5 & 16,2 & \\
\hline III & 40,9 & 21 & \\
\hline IV & 50,7 & 14,8 & \\
\hline
\end{tabular}




\begin{tabular}{lcc}
\hline Etiologia & & \\
\cline { 1 - 3 } Hipertensiva & 43,3 & 15,8 \\
\hline Chagásica & 70 & 33 \\
\hline Isquêmica & 42,8 & 22,2 \\
\hline Idiopática & 43,8 & 19,9 \\
\hline Valvar & 40 & 18,6 \\
\hline Alcoólica & 27,8 & 1,9
\end{tabular}

Tabela 4 - Análise de diferença de médias entre as variáveis selecionadas e a Self-Care Heart Failure Index, subescala manejo do autocuidado, versão brasileira ( $N=57)$. Região Metropolitana do Recife, PE, Brasil, 2016

\begin{tabular}{|c|c|c|c|}
\hline Variáveis & Média & DP & p-valor \\
\hline Gênero & & & 0,391 \\
\hline Masculino & 40,2 & 24,8 & \\
\hline Feminino & 34,8 & 21,7 & \\
\hline Escolaridade & & & 0,095 \\
\hline Analfabeto & 31 & 20,8 & \\
\hline Alfabetizado & 41,7 & 24,2 & \\
\hline Classe Funcional & & & 0,841 \\
\hline 1 & 33,3 & 11,7 & \\
\hline II & 38,1 & 21,4 & \\
\hline III & 37 & 26,1 & \\
\hline IV & 46 & 27 & \\
\hline Etiologia & & & 0,126 \\
\hline Hipertensiva & 34 & 20,1 & \\
\hline Chagásica & 72,5 & 3,5 & \\
\hline Isquêmica & 39,1 & 19,8 & \\
\hline Idiopática & 44,2 & 25,1 & \\
\hline Valvar & 38,3 & 46,5 & \\
\hline Alcoólica & 13,3 & 2,9 & \\
\hline
\end{tabular}

Tabela 5 - Análise de diferença de médias entre as variáveis selecionadas e a Self-Care Heart Failure Index, subescala confiança no autocuidado, versão brasileira ( $N=57)$. Região Metropolitana do Recife, PE, Brasil, 2016 (continua)

\begin{tabular}{|c|c|c|c|}
\hline Variáveis & Média & DP & $\mathrm{p}$-valor \\
\hline Gênero & & & 0,548 \\
\hline Masculino & 52,8 & 22,7 & \\
\hline Feminino & 56,2 & 20,2 & \\
\hline Escolaridade & & & 0,002 \\
\hline Analfabeto & 42,9 & 22 & \\
\hline Alfabetizado & 61 & 18,4 & \\
\hline Classe Funcional & & & 0,218 \\
\hline I & 70,4 & 19,4 & \\
\hline II & 51,1 & 21,1 & \\
\hline III & 51,9 & 21,4 & \\
\hline IV & 60 & 21,7 & \\
\hline
\end{tabular}




\begin{tabular}{lcc}
\hline Etiologia & & \\
\cline { 1 - 3 } Hipertensiva & 55 & 22,1 \\
\hline Chagásica & 61,2 & 15,7 \\
\hline Isquêmica & 54,6 & 23 \\
\hline Idiopática & 54,7 & 24,2 \\
\hline Valvar & 57,5 & 19,5 \\
\hline Alcoólica & 38,9 & 0
\end{tabular}

\section{- DISCUSSÃO}

Neste estudo, as médias dos escores obtidos nas escalas de manutenção, manejo e confiança no autocuidado da SCHFI, versão 6.2, foram todas abaixo de 70 pontos (Tabela 2), que é o limite mínimo de escores indicativos de autocuidado adequado.

Esses resultados são semelhantes ao estudo realizado no estado do Rio Grande do Sul, a manutenção do autocuidado $(47$; desvio-padrão- $d p=28,3)$ e a confiança no autocuidado $(58 ; d p=25,5)^{(8)}$ foram semelhantes aos deste estudo (43,3; $d p=2,5$ e 54,3; $d p=2,8$, respectivamente, conforme Tabela 2). No entanto, não ocorreu o mesmo com o manejo do autocuidado, que, no estudo mencionado, teve escore médio de $57(\mathrm{dp}=14,3)$ e neste 37,5 (dp=3,1; Tabela 2).

O conteúdo da escala de manutenção do autocuidado trata de comportamentos para manter a estabilidade fisiológica ${ }^{(4)}$. A proporção de autocuidado adequado $(3,5 \%)$, na escala manutenção do autocuidado, foi relativamente parecida com o resultado de uma avaliação realizada na cidade de São Paulo que foi $6,9 \%{ }^{(4)}$, porém em outra pesquisa realizada na Itália o valor observado foi superior $(55,26 \%)$ ao resultado desse estudo, mesmo sendo menor do que a escala classifica como adequado, mostrando uma diferença significativa ${ }^{(9)}$.

Isso pode ser explicado pela falta de conhecimento dos pacientes em relação à doença e aos cuidados adequados. A participação em programas multidisciplinares voltados para o controle dos sintomas é estratégia comumente realizada em outras realidades, diferentes da realidade da população deste estudo, o que poderia explicar os resultados em relação à manutenção do autocuidado(4).

Entre os itens da subescala manutenção do autocuidado, os escores médios mais baixos foram para os comportamentos: "Realiza alguma atividade física?", "Exercita-se por 30 minutos?" (Tabela 2). Esses achados são semelhantes aos de outros estudos, evidenciando que o baixo nível de atividade física é comum entre os pacientes com Insuficiência Cardíaca, apesar das possíveis diferenças culturais ${ }^{(4,9-10)}$.

O baixo nível de atividade física pode estar relacionado à intolerância à atividade e à exacerbação dos sintomas aos esforços, comum em pacientes com IC, e à escassez de programas para a promoção de prática de atividades físicas pelos pacientes com IC em nosso meio ${ }^{(4)}$. A evidência dos benefícios da atividade física fez do exercício físico uma importante forma de controle e tratamento da doença.

A orientação sobre o exercício a realizar deve ser individualizada de acordo com o grau da IC e a idade do paciente. Um programa domiciliar de caminhadas é uma das melhores opções para prevenir as consequências negativas, tanto fisiológicas quanto psicológicas, da inatividade. Os pacientes devem ser orientados a identificar se existe ausência ou presença de cansaço e de falta de ar para realizar as atividades do dia-a-dia e, a partir daí, iniciarem gradualmente a prática de exercício físico se não houver expressa restrição médica ${ }^{(11)}$.

A subescala manejo do autocuidado está relacionada ao comportamento dos pacientes quando ocorrem os sintomas. A proporção de pacientes com autocuidado adequado (14\%) foi igual ao resultado encontrado em um estudo realizado na cidade de São Paulo (14,7\%), superior ao encontrado em um estudo com tailandeses $(5,0 \%)^{(12)}$, mas inferior ao encontrado em amostras de italianos $(24,4 \%)$ ${ }^{(9)}$ e de chineses $(34,9 \%)^{(10)}$. 
O item "Ingerir um diurético a mais" teve um dos menores escores na escala de manejo do autocuidado (Tabela 2), o que pode ter contribuído para que o escore médio nessa escala tenha ficado muito abaixo do adequado, assim como o observado em outros estudos ${ }^{(4,9-10)}$. No hospital onde foi realizada esta pesquisa, recomendar o uso de diurético extra não é uma prática rotineira.

A proporção de pacientes com confiança no autocuidado adequado (26,3\%) foi superior ao estudo realizado em São Paulo $(19,0 \%)^{(4)}$ e em países desenvolvidos como a Itália $(21,2 \%)^{(9)}$. Acredita-se que a confiança no autocuidado tenha efeito moderador do autocuidado em diferentes desfechos como, por exemplo, na adesão.

A implementação de programas multidisciplinares com visitas domiciliares e seguimento telefônico para que o paciente reconheça e maneje adequadamente seus sintomas é uma combinação cuja eficácia para o autocuidado pode fortalecer a confiança do paciente no domínio de sua condição de saúde. Outra consideração importante é que a escala de confiança no autocuidado pode sofrer influência da desejabilidade social, o que requer cautela na interpretação dos resultados relativos a essa escala(4).

Os pacientes do sexo masculino obtiveram pontuação maior do que as mulheres nas subescalas de manutenção e manejo do autocuidado, porém o escore não teve significância $(p=0,460$ e $p=0,391$ respectivamente). Esse resultado se mostra diferente de outro estudo realizado na Itália em que o autocuidado das mulheres foi maior do que o autocuidado dos homens ${ }^{(9)}$. Isso pode ser explicado pelo fato do número de entrevistados homens ter sido superior ao número de participantes mulheres. Homens e mulheres com IC podem ter experiências diferentes e, assim, usar estratégias distintas para confrontar a doença. Considera-se que as mulheres podem ter uma melhor percepção da saúde e construir estratégias mais eficientes de enfrentamento do que os homens ${ }^{(13)}$.

Os pacientes alfabetizados tiveram melhor autocuidado em relação aos analfabetos nas 3 subescalas, mas só a escala de confiança do autocuidado tem significância $(p=0,002)$. O resultado corrobora com uma pesquisa realizada no Rio Grande do Sul, pacientes com maior escolaridade apresentaram maiores escores de autocuidado(14). Assim como em nosso estudo, quanto maior a escolaridade do paciente, maior a facilidade deste para compreender sua patologia, sinais/sintomas e maior facilidade na tomada de decisões para a promoção, recuperação e proteção da sua saúde.

Pacientes com classe funcional IV tiveram melhor autocuidado nas subescalas manutenção e manejo do autocuidado, mas não teve significância. Esse resultado difere do estudo realizado na Itália ${ }^{(9)}$, pacientes com classe funcional IV tinham um pior autocuidado nas três subescalas. Na subescala de confiança, o paciente com classe funcional I teve um bom autocuidado, já em pesquisa realizada com chineses, os pacientes que tinham classe funcional II tiveram melhor autocuidado na subescala de confiança $^{(10)}$.

Em relação à etiologia, não houve significância nos resultados das três subescalas. Não foi encontrado na literatura estudos que relacionassem a etiologia com o autocuidado do paciente com insuficiência cardíaca.

O estudo mostrou que os pacientes participantes tem um autocuidado deficiente. Este resultado sugere a necessidade de intervenções urgentes para promover o autocuidado na população.

Os enfermeiros têm papel fundamental na promoção da saúde desses pacientes, promovendo ações para que eles possam ser capacitados para o autocuidado, e assim, ter autonomia na manutenção da condição de saúde e com isso uma melhor qualidade de vida.

A avaliação do comportamento de autocuidado na IC revela-se fundamental na abordagem dos clientes com IC, para que os enfermeiros possam contribuir efetivamente para o desenvolvimento de competências cognitivas e instrumentais que facilitem a adoção de estratégias adaptativas, que contribuam para a manutenção da independência no autocuidado, num processo de capacitação e autonomia. As ações visam a promover melhorias na qualidade de vida dos pacientes, tornandoos mais ativos na tomada de decisão ao modo de viver, assumindo responsabilidades quanto aos comportamentos que levam à melhoria da sua saúde ${ }^{(11)}$.

Este estudo teve como limitação a falta do conhecimento dos pacientes sobre a doença, o que dificultou o entendimento de algumas perguntas do questionário. 
O estudo mostrou que o autocuidado neste grupo de brasileiros é inadequado, porém semelhante a outros países. Outros estudos devem ser conduzidos com a finalidade de conhecer os modelos de autocuidado na IC e identificar variáveis relevantes na resposta do autocuidado.Os resultados desta pesquisa serão úteis para o desenvolvimento de intervenções de enfermagem que possam promover e melhorar o autocuidado dessa população.

Estudos que produzam e sintetizem evidências sobre as intervenções para a manutenção e o manejo do autocuidado em pacientes com IC são necessários para orientar as decisões terapêuticas da equipe, tendo em vista a melhor qualidade de vida possível desses pacientes.

\section{REFERÊNCIAS}

1. Galvão PC da C, Gomes ET, Figueirêdo TR, Bezerra SMMS. Diagnósticos de enfermagem aplicados a pacientes com insuficiência cardíaca descompensada. Cogitare Enferm. [Internet] 2016;21(2) [acesso em 6 fev 2017]. Disponível: http://dx.doi.org/10.5380/ce.v21i2.44646.

2. de Freitas MTS, Puschel VAA. Insuficiência Cardíaca: expressões do conhecimento das pessoas sobre a doença. Rev. esc. enferm. USP. [Internet] 2013;47(4) [acesso em 8 fev 2017]. Disponível: http://dx.doi.org/10.1590/ S0080-623420130000400021.

3. Bertuzzi D, de Souza EN, Moraes MA, Mussi C, Rabelo ER. The knowledge of patients with heart failure in the homecare context: an experimental study. Online Brazilian J. Nurs. [Internet] 2012;11(3) [acesso em 6 fev 2017]. Disponível: http://www.objnursing.uff.br/index.php/nursing/article/view/3736/html.

4. da Conceição AP, dos Santos MA, dos Santos B, da Cruz DALM. Self-care in heart failure patients. Rev. LatinoAmericana Enfermagem. [Internet] 2015;23(4) [acesso em 25 jan 2017]. Disponível: http://dx.doi.org/10.1590/01041169.0288.2591.

5. Azzolin KO, Lemos DM, Lucena AF, Rabelo-Silva ER. Home-based nursing interventions improve knowledge of disease and management in patients with heart failure. Rev. Latino-Am. Enfermagem. [Internet] 2015;23(1) [acesso em 8 fev 2017]. Disponível: http://dx.doi.org/10.1590/0104-1169.0144.2523.

6. Bocchi EA, Braga FGM, Ferreira SMA, Rohde LEP, de Oliveira WA, de Almeida DR, et al. III Diretriz brasileira de insuficiência cardíaca crônica. Arq. Bras. Cardiol. 2009;93(Suppl. 1):1-71.

7. Ávila CW. Adaptação transcultural e Validação da Self-Care of heart failure index Versão 6.2 para o uso no Brasil [dissertação]. Porto Alegre: Universidade Federal do Rio Grade do Sul; 2012.

8. Ávila CW, Riegel B, Pokorski SC, Camey S, Silveira LCJ, Rabelo-Silva ER. Cross-Cultural Adaptation and Psychometric Testing of the Brazilian Version of the Self-Care of Heart Failure Index Version 6.2. Nurs. Res. Pract. [Internet] 2013;(178976) [acesso em 10 fev 2010]. Disponível: http://dx.doi.org/10.1155/2013/178976.

9. Cocchieri A, Riegel B, D'Agostino F, Rocco G, Fida R, Alvaro R, et al. Describing self-care in Italian adults with heart failure and identifying determinants of poor self-care. Eur J Cardiovasc Nurs. [Internet] 2015;14(2) [acesso em 6 fev 2017]. Disponível: http://dx.doi.org/10.1177/1474515113518443.

10. Tung HH, Chen SC, Yin WH, Cheng CH, Wang TJ, Wu SF. Self care behavior in patients with heart failure in Taiwan. Eur J Cardiovasc Nurs. [Internet] 2012;11(2) [acesso em 6 fev 2017]. Disponível: http://dx.doi.org/10.1016/j. ejcnurse.2011.02.002.

11. Pereira FAC. O Autocuidado na Insuficiência Cardíaca: tradução, adaptação e validação da european heart failure self-care behaviour scale para o contexto português [dissertação]. Porto: Instituto de Ciências Biomédicas Abel Salazar da Universidade do Porto em Ciências de Enfermagem; 2013.

12. Lee CS, Suwanno J, Riegel B. The relationship between self-care and health status domains in Thai patients with heart failure. Eur J Cardiovasc Nurs. [Internet] 2009;8(4) [acesso 10 fev 2017]. Disponível: http://dx.doi. org/10.1016/j.ejcnurse.2009.04.002. 
13. Ferreira VMP, Silva LN, Furuya RK, Schmidt A, Rossi LA, Dantas RAS. Self-care, sense of coherence and depression in patients hospitalized for descompensated heart failure. Rev. esc. enferm. USP. [Internet] 2015;49(3) [acesso em 17 jan 2017]. Disponível: http://dx.doi.org/10.1590/S0080-623420150000300005.

14. Linn AC, Azzolin K, de Souza EN. Association between self-care and hospital readmissions of patients with heart failure. Rev. bras. enferm. [Internet] 2016;69(3) [acesso em 17 jan 2017]. Disponível: http://dx.doi. org/10.1590/0034-7167.2016690312i. 